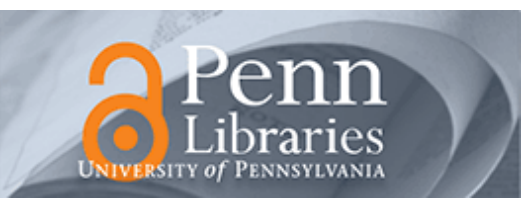

University of Pennsylvania

ScholarlyCommons

March 2004

\title{
Electronic Transport Properties of Incipient Graphitic Domains Formation in PAN Derived Carbon Nanofibers
}

\author{
Yu Wang \\ University of Pennsylvania, wangyu@seas.upenn.edu \\ Idalia Ramos \\ University of Puerto Rico \\ Rogerio Furlan \\ University of Puerto Rico \\ Jorge J. Santiago-Aviles \\ University of Pennsylvania, santiago@seas.upenn.edu
}

Follow this and additional works at: https://repository.upenn.edu/ese_papers

\section{Recommended Citation}

Yu Wang, Idalia Ramos, Rogerio Furlan, and Jorge J. Santiago-Aviles, "Electronic Transport Properties of Incipient Graphitic Domains Formation in PAN Derived Carbon Nanofibers", . March 2004.

Copyright 2004 IEEE. Reprinted from IEEE Transactions on Nanotechnology, Volume 3, Issue 1, March 2004, pages 80-85.

Publisher URL: http://ieeexplore.ieee.org/xpl/tocresult.jsp?isNumber=28578\&page=1

This material is posted here with permission of the IEEE. Such permission of the IEEE does not in any way imply IEEE endorsement of any of the University of Pennsylvania's products or services. Internal or personal use of this material is permitted. However, permission to reprint/republish this material for advertising or promotional purposes or for creating new collective works for resale or redistribution must be obtained from the IEEE by writing to pubs-permissions@ieee.org. By choosing to view this document, you agree to all provisions of the copyright laws protecting it.

This paper is posted at ScholarlyCommons. https://repository.upenn.edu/ese_papers/21

For more information, please contact repository@pobox.upenn.edu. 


\title{
Electronic Transport Properties of Incipient Graphitic Domains Formation in PAN Derived Carbon Nanofibers
}

\author{
Abstract \\ The carbon nanofibers used in this work were derived from a polyacrylonitrile (PAN)/N, N-dimethyl \\ formamide (DMF) precursor solution using electrospinning and vacuum pyrolysis techniques. Their \\ conductivity, $\sigma$, was measured at temperatures between 1.9 and $300 \mathrm{~K}$ and transverse magnetic field \\ between -9 and $9 T$. Zero magnetic field conductivity $\sigma(0, T)$ was found to increase monotonically with the \\ temperature with a convex $\sigma(0, T)$ versus T curve. Conductivity increases with the external transverse \\ magnetic field, revealing a negative magnetoresistance at temperatures between 1.9 and $10 \mathrm{~K}$, with a \\ maximum magnetoresistance of $-75 \%$ at $1.9 \mathrm{~K}$ and $9 T$. The magnetic field dependence of the \\ conductivity and the temperature dependence of the zero-field conductivity are best described using the \\ two-dimensional weak localization effect.

\section{Keywords} \\ Electronic transport, electrospinning, graphitic domains, magnetoresistance, nanofibers

\section{Comments} \\ Copyright 2004 IEEE. Reprinted from IEEE Transactions on Nanotechnology, Volume 3, Issue 1, March \\ 2004, pages 80-85. \\ Publisher URL: http://ieeexplore.ieee.org/xpl/tocresult.jsp?isNumber=28578\&page=1 \\ This material is posted here with permission of the IEEE. Such permission of the IEEE does not in any way \\ imply IEEE endorsement of any of the University of Pennsylvania's products or services. Internal or \\ personal use of this material is permitted. However, permission to reprint/republish this material for \\ advertising or promotional purposes or for creating new collective works for resale or redistribution must \\ be obtained from the IEEE by writing to pubs-permissions@ieee.org. By choosing to view this document, \\ you agree to all provisions of the copyright laws protecting it.
}




\title{
Electronic Transport Properties of Incipient Graphitic Domains Formation in PAN Derived Carbon Nanofibers
}

\author{
Yu Wang, Idalia Ramos, Rogerio Furlan, and Jorge J. Santiago-Avilés
}

\begin{abstract}
The carbon nanofibers used in this work were derived from a polyacrylonitrile (PAN)/N, N-dimethyl formamide (DMF) precursor solution using electrospinning and vacuum pyrolysis techniques. Their conductivity, $\sigma$, was measured at temperatures between 1.9 and $300 \mathrm{~K}$ and transverse magnetic field between -9 and $9 T$. Zero magnetic field conductivity $\sigma(0, T)$ was found to increase monotonically with the temperature with a convex $\sigma(0, T)$ versus $T$ curve. Conductivity increases with the external transverse magnetic field, revealing a negative magnetoresistance at temperatures between 1.9 and $10 \mathrm{~K}$, with a maximum magnetoresistance of $-75 \%$ at $1.9 \mathrm{~K}$ and $9 T$. The magnetic field dependence of the conductivity and the temperature dependence of the zero-field conductivity are best described using the two-dimensional weak localization effect.
\end{abstract}

Index Terms-Electronic transport, electrospinning, graphitic domains, magnetoresistance, nanofibers.

\section{INTRODUCTION}

C ARBON nanofibers, like other quasi-one-dimensional nanostructures such as nanowires, nanotubes and molecular wires, are receiving increased attention. This is due to their potential application in a multiplicity of fields, such as high-temperature catalysis, heat-management materials in aircraft, and filters for separation of small particles from gas or liquid. Of more importance to us, there is a possibility of its use as building blocks for bottom-up assembly applications in nanoelectronics and photonics [1]-[3]. Carbon fibers are usually produced by spinning from organic precursor fibers or by chemical vapor deposition (CVD). While the spinning method can only produce microscale carbon fibers, CVD can synthesize carbon fibers with diameters from several microns down to less than $100 \mathrm{~nm}$ [4], [5]. However, CVD involves a complicated process and high cost. Electrostatic generation, or electrospinning technique, invented in the 1930s [6], recently gained renewed interest because it can spin a variety of ultrafine polymer fibers in a micro- or even nanoscale at low cost [7]. By simply pyrolyzing electrospun ultrafine polymer fibers, with a

Manuscript received June 14, 2003; revised November 21, 2003. This work was supported in part by the National Science Foundation, under (NSF)-DMR-9872689. This paper was presented in part at the Symposium of Microtechnologies for the New Millenium, Nanotechnology Conference, Gran-Canaria, Spain, May 2003.

Y. Wang and J. J. Santiago-Avilés are with the Department of Electrical Engineering, University of Pennsylvania, Philadelphia, PA 19104 USA.

I. Ramos and R. Furlan are with the Department of Physics and Electronics, University of Puerto Rico at Humacao, Humacao, 00791 Puerto Rico (e-mail: iramos@www.uprh.edu).

Digital Object Identifier 10.1109/TNANO.2004.824036 subsequent heat treatment, Chun et al. [8] and the authors [9] have obtained carbon nanofibers.

It is well known that the electrical conductivity of pyrolytic graphite increases with temperature. Such temperature dependence was at first explained by the simple two-band (STB) model [10]. The STB model also predicts a level off of the conductivity at a very low temperature. However, recent experimental results show that the conductivity of carbon fibers is very sensitive to temperature at very low values $(T<10 \mathrm{~K})$ [11]-[13]. Such anomaly has been attributed to weak electron localization [13], electron-electron interaction [13], the Kondo effect [13], and hopping mechanism [14], all of which show very weak effects unless evaluated at very low temperatures. As to the overall temperature dependence of conductivity, two-dimensional (2-D) weak localization, hopping and tunneling [15] mechanisms have been put forward as possible explanations.

Although classical electron transport theory predicts an increase of electrical resistance in the presence of a magnetic field [16], Mrozowski and Chaberski found a decrease of resistance with magnetic field, or negative magnetoresistance, in partially ordered (pregraphitic) carbons [17]. Since then, negative magnetoresistance has been found not only in poorly graphitized bulk carbon [18] and carbon thin film [19], but also in carbon fibers, irrespective of whether the carbon fibers were derived from PAN [13], benzene [20], pitch-derived [11], or CVD [21]. The most commonly accepted model accounting for the negative magnetoresistance was Bright's model [22]. This model attributes the resistance decrease to the increase of the density of the states and carrier density with magnetic field, arising from the formation of Landau levels. However, the Bright model cannot account for all of the observed phenomena, including the strong temperature dependence of magnetoresistance below liquid-helium temperature, and the absence of magneto-resistance saturation at high-magnetic field. Then, Bayot et al. [23], [24] explained the effect using a weak-localization mechanism, which results as a consequence of any small disorder in the electronic system. The weak-localization effects in pregraphitic carbon fibers are due to their turbostratic phase structure, in prior 2-D.

\section{EXPERIMENTAL}

The precursor is commercial polyacrylonitrile (PAN) and $\mathrm{N}$, $\mathrm{N}$-dimethyl formamide (DMF) solution, in a ratio of $600 \mathrm{mg}$ PAN to $10 \mathrm{ml}$ DMF. The substrates, silicon wafers with a 


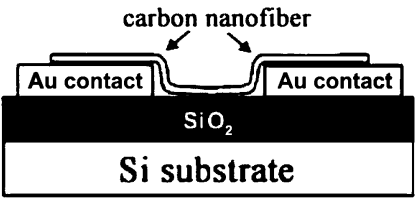

Fig. 1. Schematic of carbon nanofiber deposited on substrate.

150-nm-thick film of silicon oxide, were lithography patterned with $1 \times 1 \mathrm{~mm}^{2}$ gold contact array. A homemade electrospinning setup discussed elsewhere [9], was used to spin single precursor fibers between two isolated gold contacts (Fig. 1). The as-spun PAN fibers were pyrolyzed and heat treated at $1273 \mathrm{~K}$ for $30 \mathrm{~min}$ in a vacuum of $10^{-6}$ torr in a Brew model 466-S vacuum furnace equipped with a mesh-heating element. The processed fibers were characterized using a Renishaw Raman microspectrometer at room temperature with a green laser (wavelength $=514 \mathrm{~nm}$ ) as the exciting radiation.

We used a two point-probe setup to continuously monitor the conductance $(G)$ in the temperature range between 300 and $1.9 \mathrm{~K}$, back and forth, without any applied magnetic field, using Model 6000 Physical Properties Measurement System by Quantum, Inc., equipped with a Keithley 237 high-voltage source measurement unit. Conductance was also measured at $1.9,3.0,5.0$, and $10.0 \mathrm{~K}$ while the applied magnetic field, perpendicular to the fiber, was increased or decreased continuously between -9 and $9 T$ twice. To suppress possible heating effects, the total measuring power was limited to $5 \mathrm{nW}$. According to a previous analysis [25], the contact resistance is much less than that of the nanofiber itself. The length $L$ and cross section area $S$ of the fibers were measured using an optical microscope and a scanning probe microscope (SPM) operated in tapping mode. Their details have been reported elsewhere [25]. The conductivity was finally determined using $\sigma=$ GL/S.

\section{RESULTS}

Fig. 2 shows SPM height image of vacuum pyrolyzed and heat-treated carbon nanofiber and its apparent average cross section profile, from which the vertical and horizontal diameters of the fiber $(b, a)$ and, therefore, its cross section area $(S)$, were determined [25]: $a=120 \pm 10 \mathrm{~nm}, b=70 \pm 5 \mathrm{~nm}$, $S=6600 \pm 100 \mathrm{~nm}^{2}$. Fig. 3 shows the Raman spectrum of the same carbon nanofibers whose $G$ and $D$ peaks, centered at 1371 and $1588 \mathrm{~cm}^{-1}$, attest to the coexistence of disorder and graphitic carbon in the nanofibers. From the ratio of the integrated intensity of $D$ peak to $G$ peak, the inplane graphitic crystallite size $L_{\mathrm{a}}$ was estimated to be $2.47 \pm 0.08 \mathrm{~nm}$ [9].

Fig. 4 shows $\sigma$ as a smooth and monotonic increasing function of $T$, showing apparently semiconducting nature. Moreover, $\sigma(0, T)$ versus $T$ curve is convex, in other words, $\mathrm{d} \sigma(0, T) / \mathrm{dT}$ decreases with the increase of $T$.

Fig. 5(a) shows the magnetoresistence MR, defined as $\mathrm{MR}=\rho(B) / \rho(0)-1$, and the magnetoconductivity $\mathrm{MC}=\sigma(B) / \sigma(0)-1$ of the fibers at temperatures of 1.9, 3.0, 5.0, and $10 \mathrm{~K}$ with magnetic field $\mathrm{B}$ from -9 to $9 \mathrm{~T}$. At all four investigated temperatures, the MR is negative. Its magnitude increases with an increase in $\mathrm{B}$ and a decrease in $\mathrm{T}$. It is noteworthy that $\mathrm{MR}=-0.75$ at $T=1.9 \mathrm{~K}$ and $B=9 \mathrm{~T}$, one of the largest negative magnetoresistance known to the authors. Since $|\mathrm{MR}|$ is quite large, $\mathrm{MR} \approx(\mathrm{MC})^{-1}$ is not always valid.

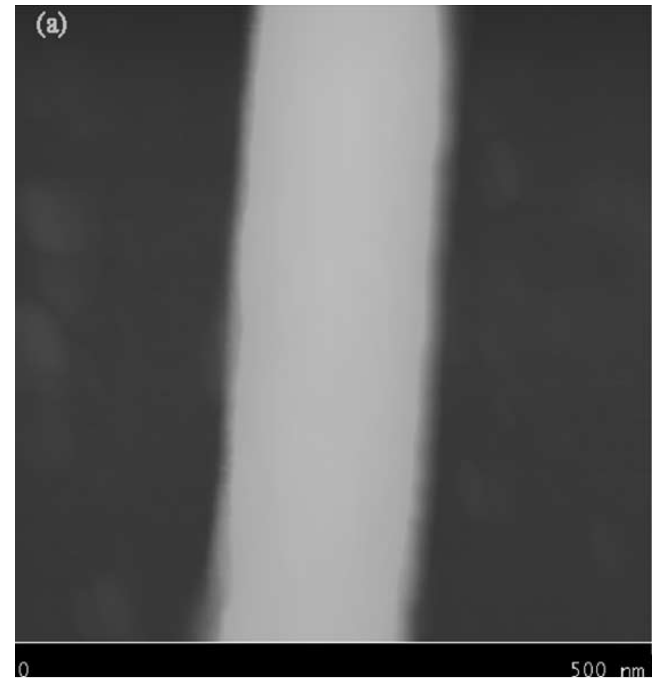

(b) $\mathrm{nm}$

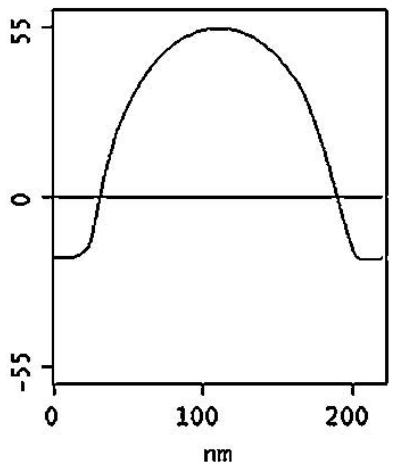

Fig. 2. (a) SPM height image of Carbon fiber and (b) average cross section profile.

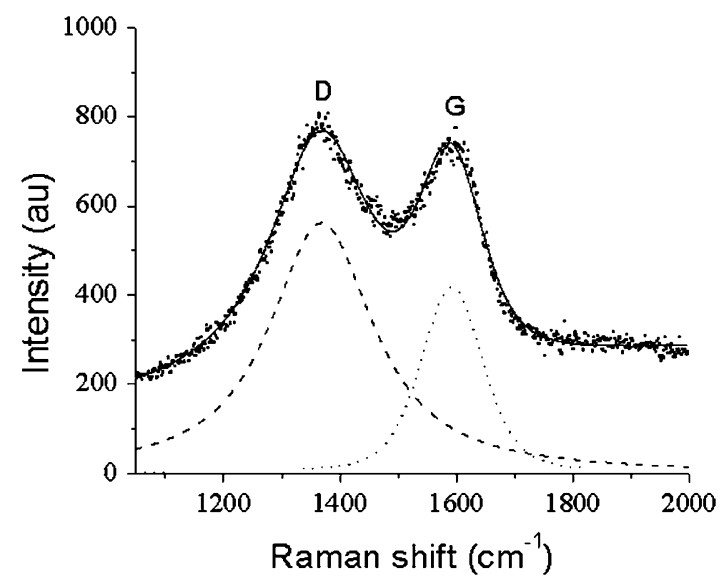

Fig. 3. Raman spectrum of the pyrolized fiber.

\section{DISCUSSION}

\section{A. Magnetic Field Dependence of Magnetoresistance}

For most electronic systems exhibiting 2-D weak localization, the physical origin of the 2-D character is relatively easy to understand. In carbon fibers, however, the origin is a priori not obvious. Bright attributed the origin to the turbostratic nature of the samples, which should have an electronic structure nearly the same as that of 2-D graphite. In the 2-D regime, the correction to the sheet conductance $G$ produced with the 


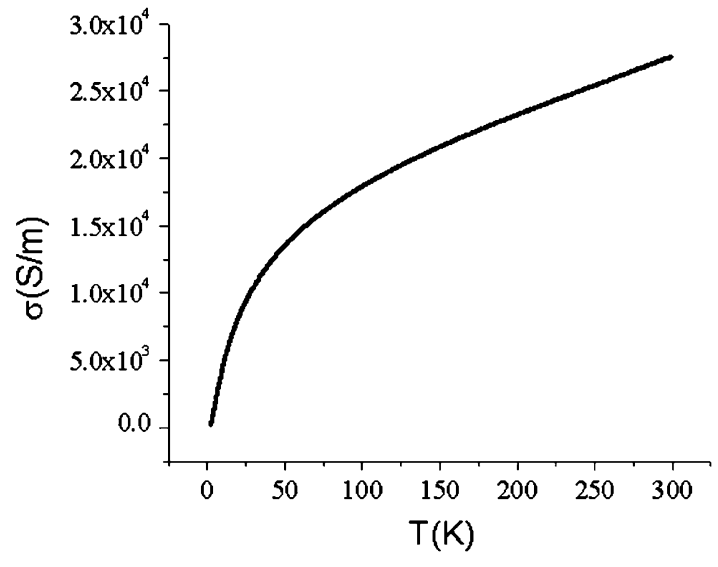

Fig. 4. Temperature dependence of the conductivity of carbon fibers.
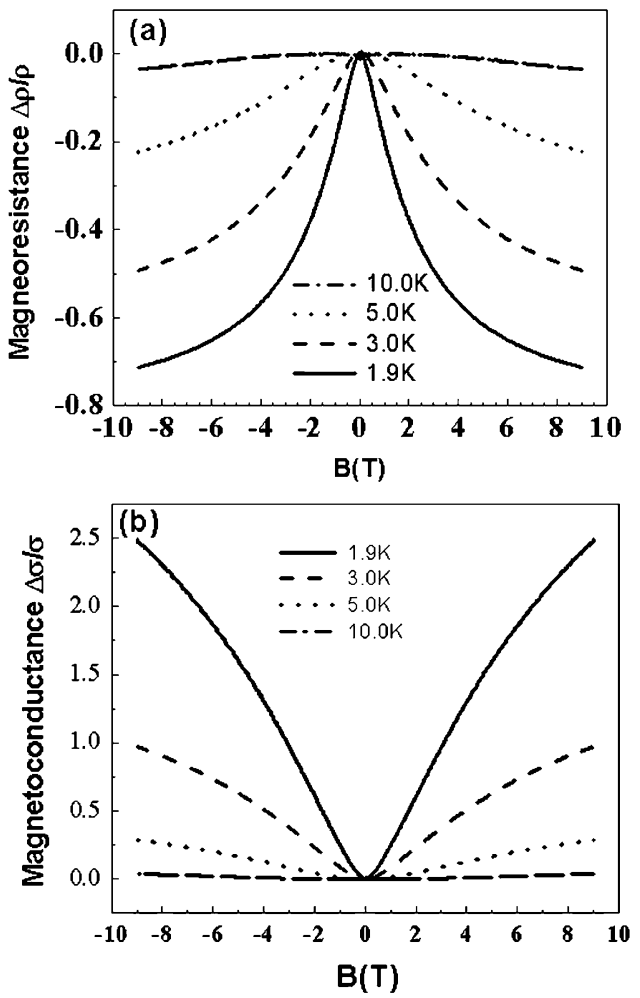

Fig. 5. (a) Large negative magnetoresistance and (b) positive magnetoconductance of carbon fibers with magnetic field from -9 to $9 \mathrm{~T}$.

magnetic field B perpendicular to the plane of the 2-D carrier system is given by the following expression [23], [24], [26]:

$$
\begin{aligned}
\mathrm{G}(B, T)=\mathrm{G}_{\infty}+ & \frac{e^{2}}{\pi \hbar}\left[\frac{3}{2} \Psi\left(\frac{1}{2}+\frac{B_{2}}{B}\right)\right. \\
& \left.-\Psi\left(\frac{1}{2}+\frac{B_{1}}{B}\right)-\frac{1}{2} \Psi\left(\frac{1}{2}+\frac{B_{3}}{B}\right)\right]
\end{aligned}
$$

where $B_{1}=B_{0}+B_{\text {s.o. }}+B_{s}, B_{2}=B_{i}(T)+(4 / 3) B_{\text {s.o. }}+$ $(2 / 3) B_{\mathrm{s}}, B_{3}=B_{\mathrm{i}}(T)+2 B_{\mathrm{s}}, G_{\infty}$ is the sheet conductance at infinite magnetic field, or as calculated in the classical Boltzmann formulation of the transport theory; $\Psi$ is digamma function, and $B_{\mathrm{k}}(k=0, \mathrm{i}, \mathrm{s}$, s.o. $)$ represents the characteristic field associated with the scattering mechanism $k \mathrm{k}$ standing for elastic scattering (0), inelastic scattering (i), magnetic impurity scattering (s) and spin-orbit coupling (s.o.). Therefore, the magnetoconductance takes the form

$$
\begin{aligned}
\mathrm{MC}=\eta(T)\left[\frac{3}{2} \Psi(\right. & \left.\frac{1}{2}+\frac{B_{2}}{B}\right)-\Psi\left(\frac{1}{2}+\frac{B_{1}}{B}\right) \\
& \left.-\frac{1}{2} \Psi\left(\frac{1}{2}+\frac{B_{3}}{B}\right)-\ln \frac{B_{2}^{3 / 2}}{B_{1} B_{3}^{1 / 2}}\right]
\end{aligned}
$$

where $\eta(T)=\left(e^{2} / \pi h G(0, T)\right)$.

Given a temperature $T$, (2) contains only four unknown parameters: $\eta(T), B_{1}, B_{2}$, and $B_{3}$. They can be derived from a nonlinear fitting. The results show that $B_{2}$ and $B_{3}$ have very close fitting values. In fact, their difference is less than their respective fitting errors. This indicates that both $B_{\mathrm{s} . \mathrm{o}}$ and $B_{s}$ are very small, i.e., both magnetic impurity scattering and spin-orbit coupling are very weak, in our sample. For simplicity, we assume $B_{\text {s.o }} \approx B_{s} \approx 0$, and $B_{1}=B_{0}, B_{2}=B_{3}=B_{\mathrm{i}}(T)$. Then, (2) can be simplified as

$$
\mathrm{MC}=\eta(T)\left[\Psi\left(\frac{1}{2}+\frac{B_{2}}{B}\right)-\Psi\left(\frac{1}{2}+\frac{B_{1}}{B}\right)-\ln \frac{B_{2}}{B_{1}}\right] .
$$

Nonlinear curve fitting using (3) and Mathematica software (shown in Fig. 6) showed that $B_{\mathrm{i}}$ increases with T, i.e., the inelastic scattering intensifies when the temperature increases.

\section{B. Temperature Dependence of Zero Field Conductivity}

In parallel to the modified STB model [27], which accounts for the conductivity phenomenologically, several other models can explain the temperature dependence of the conductivity, namely:

1) 2-D weak localization model: According to 2-D weak localization model [28]-[30]

$$
\sigma(T)=\sigma(0)+\frac{e^{2}}{2 \pi^{2} \hbar} \ln \left[1+\left(\frac{T}{T_{C}\left(B, \tau_{s}\right)}\right)^{p}\right]
$$

where $\sigma(0)$ and $T_{C}\left(B, \tau_{s}\right)$ are two constants. If (4) is used to fit the temperature dependence of the zero field conductivity, we obtain, $\sigma(0)=0$, and $\mathrm{p}=1.15$ (Fig. 7). The value $\mathrm{p}=1.15$ is in agreement with the previous results of $\mathrm{p} \approx 1.00$ in carbon microfibers [23] and multiwalled carbon nanotube [30]. It indicates that the dominating inelastic scattering mechanism is likely to be disorder enhanced electron-electron scattering in 2-D system.

2) Variable range hopping model: This phenomenon occurs in highly disordered materials because the distribution of energy states makes it more favorable to hop to a distant empty state of nearly the appropriate energy than to a nearby empty state that has a much higher energy level. Because of the high resistivity and lack of long-range order of the fiber, one would expect that the electrical conduction would result from a hopping mechanism. The dimensionality enters the equation during the summing of the available states. For a $d$-dimensional system [31]

$$
\sigma(T)=A \exp \left[-\frac{B}{T^{1 /(d+1)}}\right]
$$



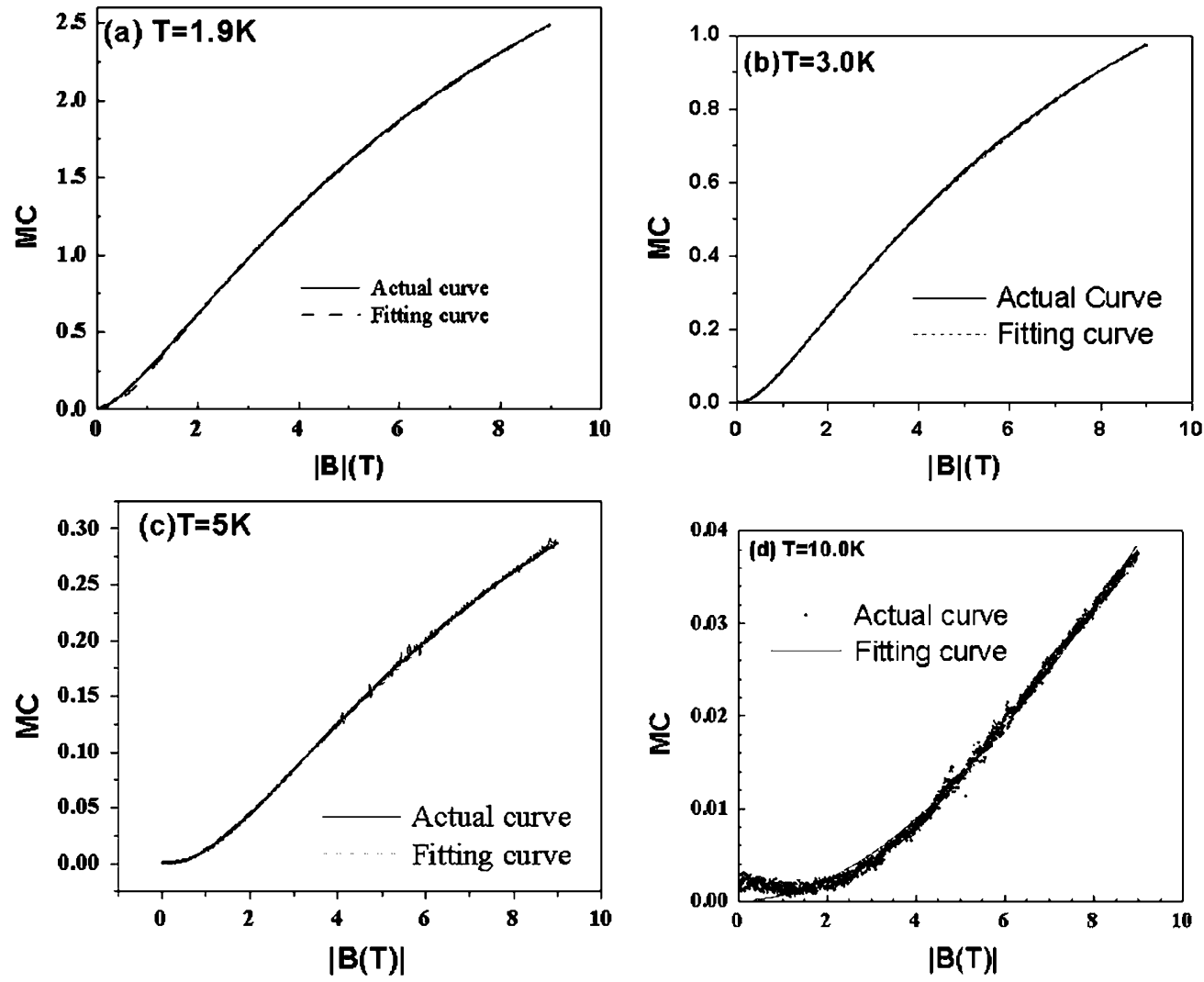

Fig. 6. Comparison of magnetoconductance fitting curves [using (3)] with experimental curves.

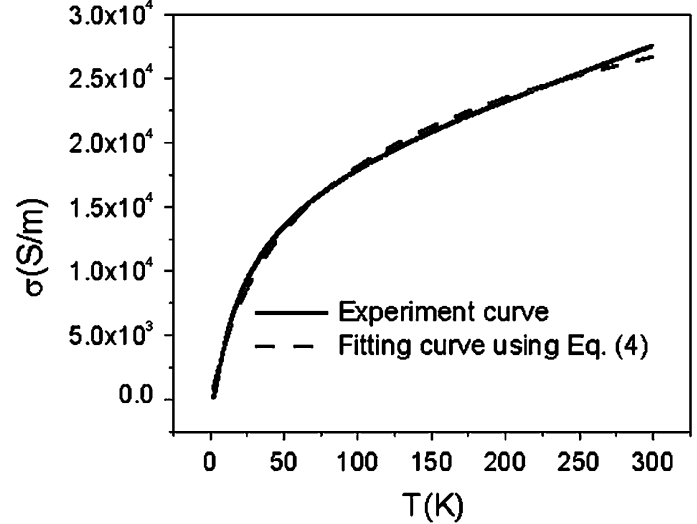

Fig. 7. Fitting of conductivity curve to (4).

where $d=1,2,3$, and $A, B$ are two constants. Since there is controversy about the dimensionality of the carbon fibers with respect to variable range hopping, a good three-parameter fit to all the data was found by least square fitting of the data to (5) (Fig. 8). The fitting results $d=0.295$ indicates that the dimensionality of hopping lies between 2 and 3 , or that $d=2$ and $d=3$ coexist.

3) Tunneling between conducting particles model: The tunneling model was developed for metallic particles imbedded in a highly resistive matrix but the only essential feature of the metallic particles is that the conduction electrons therein are delocalized. As revealed by XRD

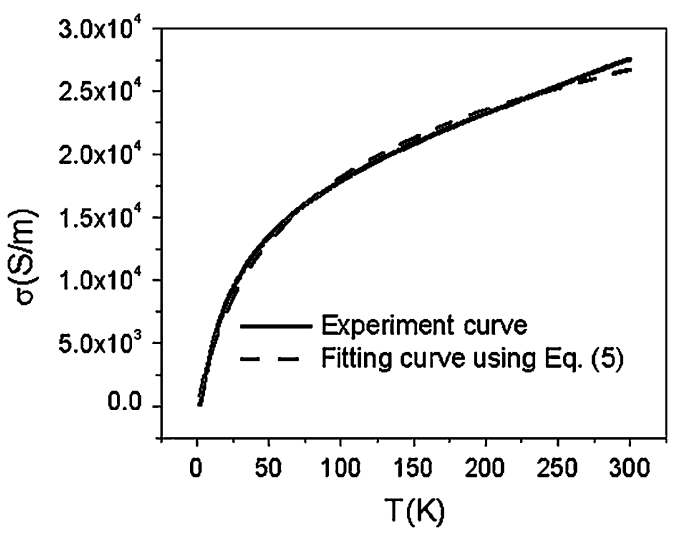

Fig. 8. Fitting of conductivity curve to (5).

and Raman spectra, the graphite domains within our carbon nanofibers have a size of 1 to $2 \mathrm{~nm}$ [9]. Since electrons on the small fully carbonized basic units fit this criterion, it is not unreasonable that the model may apply to the investigated fibers. For dc conduction, electron tunnel between the charging centers imbedded in a highly resistive matrix of totally disordered carbon might be plausible. When the electric field is low, the conduction is ohmic resulting in thermally activated charge carriers hopping to the nearest neighbor charging center. For high-electric fields, the conduction is highly nonohmic and is the result of field-induced tunneling. 


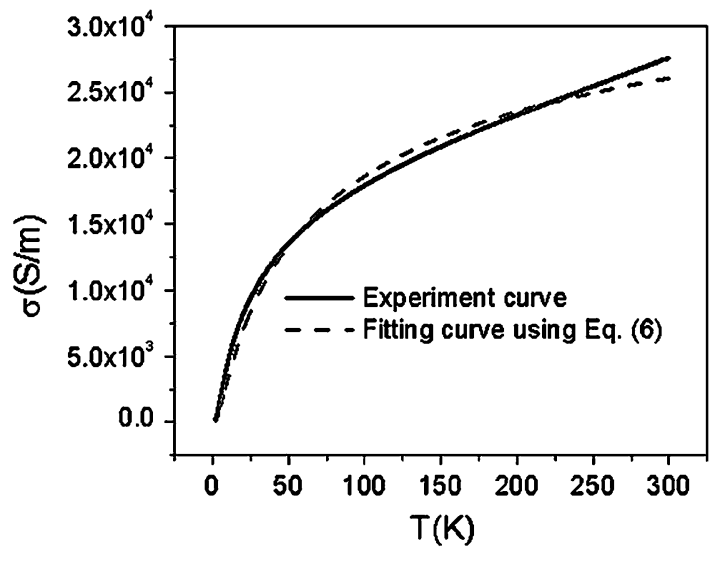

Fig. 9. Fitting of conductivity curve to (6).

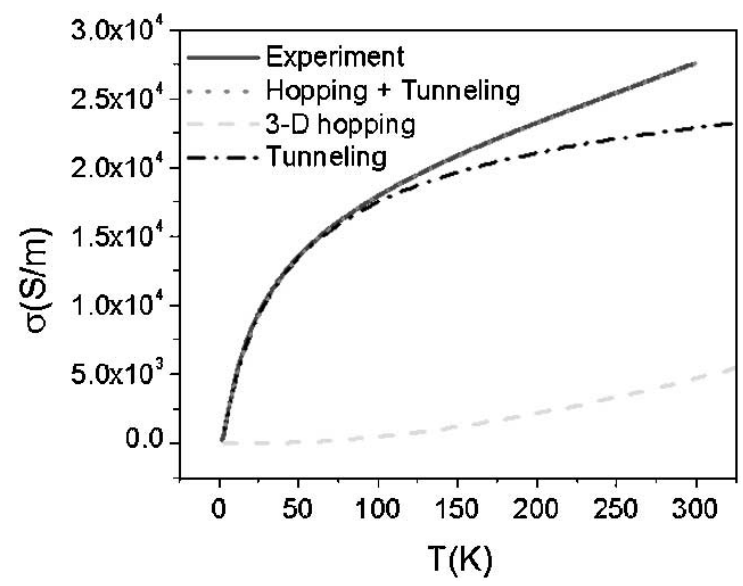

Fig. 10. Fitting of conductivity curve to (7).

The conductivity in this model has the same form as 1-d variable range hopping [15]

$$
\sigma(T)=A_{1} \exp \left[-\frac{B_{1}}{T^{1 / 2}}\right]
$$

where $A_{1}$ and $B_{1}$ are two constants. If the $\sigma(T)$ versus $T$ curve is fit to (6) [Fig. 9], we obtain $A_{1}=41172 \mathrm{~S} / \mathrm{m}, B_{1}=$ $7.92617 \mathrm{~K}^{1 / 2}$. It seems reasonable to try to fit the data to a model in which 3-D variable range hopping and tunneling between domains in which the electrons are delocalized, coexist. The conductivity for such a case is

$$
\sigma(T)=A \exp \left[-\frac{B}{T^{1 /(d+1)}}\right]+A_{1} \exp \left[-\frac{B_{1}}{T^{1 / 2}}\right] .
$$

Fig. 10 shows the results of fitting the data to (7). The fitting is excellent. The figure also shows the curves for the two components. They indicate that the conduction is mostly undertaken by a tunneling mechanism. It increases convexly with the temperature, the hopping mechanism accounting for only a small fraction of the total (its contribution to the conductivity increases concavely with the temperature). As such, the hopping mechanism can be excluded from the main transport mechanism(s) in the investigated carbon fiber.
Since both (4) and (6) fit the experimental $\sigma$ versus $T$ curve quite well, the fitting alone seems not enough to determine whether the main transport mechanism is 2-D weak localization effect, or the tunneling mechanism. However, the good description of the $T$ - and $B$ - dependence of the large MR that can be done using 2-D weak localization effect indicates that the same effect is mainly responsible for the $T$-dependence of the conductivity. This harmonizes with the low-electrical field setup during the conductance measurement. The voltage applied between the two conducting pads, separated by a distance of $1 \mathrm{~mm}$, is $0.03-0.6 \mathrm{~V}$. So, the average electrical field in the carbon nanofiber between the two pads is $30-600 \mathrm{~V} / \mathrm{m}$, not strong enough for the tunneling mechanism to dominate.

\section{CONCLUSION}

Partially graphitized carbon nanofibers were synthesized from PAN/DMF solution using electrospinning and subsequent vacuum pyrolysis technique. Their zero magnetic field conductivity and magnetoconductivity were measured using a two-probe method. Large negative magnetoresistance $(-75 \%)$ was found at $1.9 \mathrm{~K}$ and $9 \mathrm{~T}$. The temperature dependence of zero magnetic field conductivity, temperature and magnetic field dependence of the large magnetoresistance were best described using the 2-D weak localization effect.

\section{ACKNOWLEDGMENT}

The authors express their gratitude to Dr. J. Kikawwa, Physics Department, University of Pennsylvania, for his help with the conductivity measurements.

\section{REFERENCES}

[1] S. Mrozowski, "Specific-heat anomalies and spin-spin interactions in carbons-Review," J. Low Temp. Phys., vol. 35, no. 3/4, pp. 231-297, 1979.

[2] J. Hu, T. W. Odom, and C. M. Lieber, "Chemistry and physics in one dimension: Synthesis and properties of nanowires and nanotubes," Acc. Chem. Res., vol. 32, pp. 435-445, 1999.

[3] X. Duan, Y. Huang, J. Wang, and C. M. Lieber, "Indium phosphid nanowires as building blocks for nanoscale electronic and optoelectronic devices," Nature, vol. 409, pp. 66-69, Jan. 2001.

[4] O. P. Bahl, Z. Shen, J. G. Lavin, and R. A. Ross, "Manufacture of carbon fibers," in Carbon Fibers, 3rd ed, J.-B. Donnet, Ed. New York: Marcel Dekker, 1998, pp. 1-84.

[5] M. Endo, Y. A. Kim, T. Takeda, S. H. Hong, T. Matusita, T. Hayashi, and M. S. Dresselhaus, "Structural characterization of carbon nanofibers obtained by hydrocarbon pyrolysis," Carbon, vol. 39, pp. 2003-2010, Nov. 2001.

[6] A. Formhals, "Process and Apparatus for Preparing Artificial Threads," U.S. Pat. 1975 504, Oct. 2, 1934.

[7] J. Doshi and D. H. Reneker, "Electrospinning process and application of electrospun fibers," J. Electr., vol. 35, pp. 151-156, Aug. 1995.

[8] D. H. Renker and I. Chun, "Nanometer diameter fibers of polymer, produced by electrospinning," Nanotechnology, vol. 7, pp. 216-223, Sept. 1996.

[9] Y. Wang, S. Serrano, and J. J. Santiago-Aviles, "Raman characterization of carbon nanofibers prepared using electrospinning," Synthetic Metals, vol. 138, pp. 423-427, 2003.

[10] C. A. Klein, "STB model and transport properties of pyrolytic graphites," J. Appl. Phys., vol. 35, no. 10, pp. 2947-2957, 1964.

[11] A. A. Bright and L. S. Singer, "Electronic and structural characteristics of carbon fibers from mesophase pitch," Carbon, vol. 17, pp. 59-69, 1979.

[12] I. L. Spain, K. J. Volin, H. A. Goldberg, and I. Kalnin, "Electronic properties of pan-based carbon fibers I," J. Phys. Chem. Solids, vol. 44, no. 8, pp. 839-849, 1983. 
[13] Y. Koike and T. Fukase, "Anomalous electrical conduction in carbon fibers at low temperatures," Solid-State Commun., vol. 62, no. 6, pp. 499-502, May 1987.

[14] D. F. Baker and R. H. Bragg, "One-dimensionality in glassy carbon," Phys. Rev. B, vol. 28, no. 4, pp. 2219-2221, Aug. 1983.

[15] B. Abeles, P. Sheng, M. D. Coutts, and Y. Arie, "Structural and electrical properties of granular metal-films," Adv. Phys., vol. 24, no. 3, pp. 407-16, 1975.

[16] E. H. Putley, The Hall Effect and Related Phenomena, London, U.K.: Butterworth, 1960, pp. 66-97.

[17] S. Mrozowski and A. Chaberski, "Hall effect and magnetoresistivity in carbons and polycrystalline graphites," Phys. Rev., vol. 104, no. 74, pp. 74-83, Oct. 1956.

[18] Y. Hishiyama, "Negative magnetoresistance in soft carbons and graphite," Carbon, vol. 8, pp. 259-269, 1976

[19] A. Faißt and H. V. Löhneysen, "Electrical resistance and magnetoresistance of pyrocarbon at low temperatures in fields up to 14 tesla," Carbon, vol. 40, pp. 321-327, 2002

[20] M. Endo, Y. Hishiyama, and T. Koyama, "Magnetoresistance effect in graphitizing carbon fibers prepared by benzene decomposition," J. Phys. D, Appl. Phys., vol. 15, pp. 353-363, 1982.

[21] M. Fuji, M. Matsui, S. Motojima, and Y. Hishikawa, "Magnetoresistance in carbon micro-coils obtained by chemical vapor deposition," Thin Solid Films, vol. 409, pp. 78-81, 2002.

[22] A. Bright, "Negative magnetoresistance of pregraphitic carbons," Phys. Rev. B, vol. 20, no. 12, pp. 5142-5149, Dec. 1979.

[23] V. Bayot, L. Piraux, J.-P. Michenaud, and J.-P. Issi, "Weak localization in pregraphitic carbon fibers," Phys. Rev. B, vol. 40, no. 6, pp. 3514-3523, Aug. 1989.

[24] V. Bayot, L. Piraux, J. P. Michenaud, J. P. Issi, M. Lelaurain, and A Moore, "Two-dimensional weak localization in partially graphitic carbons," Phys. Rev. B, vol. 41, no. 17, pp. 11770-11 778, June 1990

[25] Y. Wang, J. J. Santiago-Avilés, R. Furlan, and I. Ramos, "Pyrolysis temperature and time dependence of electrical conductivity evolution for electrostatically generated carbon nanofibers," IEEE Trans. Nanotechnol., vol. 2, pp. 39-43, Mar. 2003.

[26] R. Rosenbaum, "Superconducting fluctuations and magnetoconductance measurements of thin films in parallel magnetic fields," Phys. Rev. B, vol. 32, no. 4, pp. 2190-2199, Aug. 1985.

[27] Y. Wang and J. J. Santiago-Avilés, "Large negative magnetoresistance and two-dimensional weak localization in carbon nanofiber fabricated using electrospinning," J. Appl. Phys., vol. 94, no. 3, pp. 1721-1727, 2003.

[28] P. A. Lee and T. V. Ramakrishnan, "Disordered electronic system," Rev. Modern Phys., vol. 57, no. 2, pp. 287-337, 1985.

[29] G. Bergmann, "Weak localization in thin films a time of flight experiment with conduction electrons," Phys. Rep., vol. 107, no. 1, pp. 1-58, May 1984.

[30] L. Langer, V. Bayot, E. Grivei, J.-P. Issi, J. P. Heremans, C. H. Olk, L. Stockman, C. Van Haesendonck, and Y. Bruynseraede, "Quantum transport in a multiwalled carbon nanotube," Phys. Rev. Lett., vol. 76, no. 3, pp. 479-482, Jan. 1996.

[31] F. Mott and E. A. Davis, Electronic Processes in Non-Crystalline Materials Clarendon, New York, 1979.

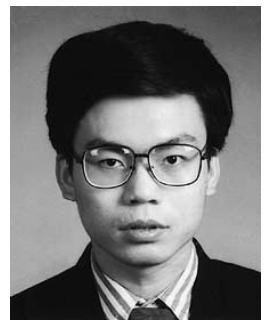

Yu Wang received the B.S. degree in materials science and engineering from Shanghai Jiaotong University, China, in 1988, and the M.S. and Ph.D. degrees in electrical engineering from the University of Pennsylvania, Philadelphia, in 2001 and 2003, respectively.

His current research interest is nanotechnology.

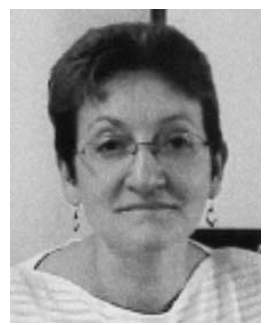

Idalia Ramos is an Associate Professor in the Department of Physics and Electronics, University of Puerto Rico, Humacao. Her research is in the areas of materials and implementation of micro-elecro-mechanical devices, micro- and nano-fibers. She is also the Program Director of two National Science Foundation funded programs: a Collaborative to Integrate Research and Education between the University of Puerto Rico and the University of Pennsylvania and the ADVANCE Institutional Transformation, a program to change the status of women in the science faculty at the University of Puerto Rico, Humacao.

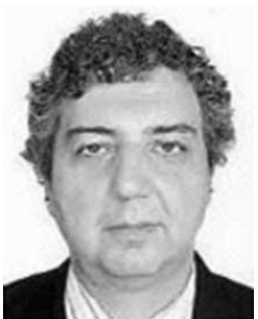

Rogerio Furlan received the electrical engineering degree from the Faculdade de Engenharia Industrial (FEI), Brazil, in 1982, and the M.S. and Ph.D. degrees in electrical engineering from the University of Sao Paulo, Brazil, in 1985 and 1990, respectively.

From 1988 to 2001, he was a Faculty Member at the Polytechnic School of University of Sao Paulo, where he became an Associate Professor in 1998. In 1981. he became a Member of the Laboratory of Integrated Systems, where he coordinated the Metallization Group from 1984 to 1989 and the Microstructures Group from 1996 to 2001, also at the University of Sao Paulo. In August 2001, he became an Associate Professor of the Physics and Electronics Department of the University of Puerto Rico at Humacao. His primary research interests include micro and nanofibers and microfluidic devices.

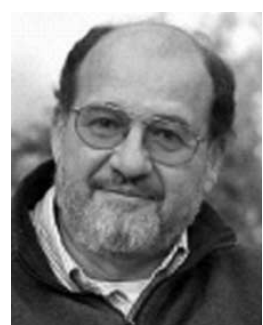

Jorge J. Santiago-Aviles is currently an Associate Professor of electrical and system engineering at the University of Pennsylvania, Philadelphia. He research work has been in II-VI semiconducting compounds for LEDs, carbon and graphite composites, silicon sensors and actuators, electroceramic devices and LTCC tape based sensing and actuating devices. His research interest includes the interface between electronic devices and materials science. Currently, his interests are focused on the field of nanostructures and nanomaterials. 